\title{
OSCILLAIORY BEHAVIOR OF NONLINEAR DIFFERENTIAL EQUATIONS WITH DEVIATING ARGUMENTS
}

\author{
S.R. GRAce AND B.S. LaLLI
}

New oscillation criteria for nonlinear differential equations with deviating arguments of the form

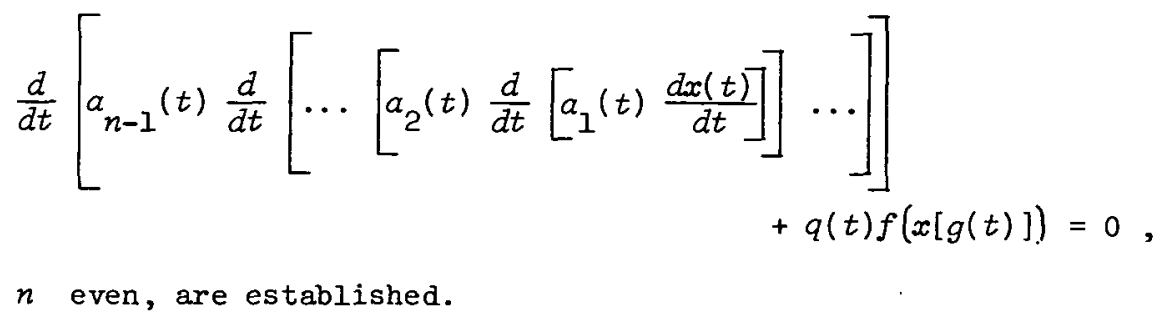

\section{Introduction}

Recently Kamenev [4] considered the linear equation

$$
\ddot{x}+q(t) x=0 \quad\left(\cdot=\frac{d}{d t}\right),
$$

where $q$ is a continuous real-valued function on the interval $\left[t_{0}, \infty\right)$ without any restriction on its sign, and proved that the condition

$$
\lim _{t \rightarrow \infty} \sup \frac{1}{t^{m-1}} \int_{t_{0}}^{t}(t-s)^{m-1} q(s) d s=\infty \text {, }
$$

for some integer $m \geq 3$, is sufficient so that all the solutions of $\left(\alpha_{1}\right)$

Received 18 September 1984.

Copyright Clearance Centre, Inc. Serial-fee code: 0004-9727/85 $\$ \mathrm{~A} 2.00+0.00$. 
are oscillatory. His criterion includes as a special case the well-known Wintner result of [8]. Kamenev's criterion has been extended in various directions by Philos $[6,7]$ and Yeh [9, 10]. The present authors [1, 2] discussed this criterion for general functional equations of the form

$$
x^{(n)}+q(t) f(x[t], x[g(t)]\}=0, n \text { even, }
$$

and

$\left(\alpha_{3}\right) \quad\left(a(t) x^{(n-1)}\right)^{*}+p(t)\left|x^{(n-1)}\right|_{x^{(n-1)}}+q(t) f(x[g(t)]\}=0$,

$$
n \text { even and } \beta \geq 0 \text {, }
$$

with $p$ and $q$ nonnegative continuous functions on the interval $\left[t_{0}, \infty\right)$.

The purpose of this paper is to extend some of the previously mentioned results and obtain new oscillation criteria for the equation

$$
L_{n} x(t)+q(t) f(x[g(t)])=0, n \text { even, }
$$

where

$$
L_{0} x(t)=x(t), \quad L_{k} x(t)=a_{k}(t)\left(L_{k-1} x(t)\right)^{\bullet} \text {, for } k=1,2, \ldots, n,
$$

with $a_{0}(t)=a_{n}(t)=1$.

\section{Main results}

Consider the equation

$$
L_{n} x(t)+q(t) f(x[g(t)])=0, n \text { even, }
$$

where

$$
L_{0} x(t)=x(t), \quad L_{k} x(t)=a_{k}(t)\left(L_{k-1} x(t)\right)^{\circ}, k=1,2, \ldots, n,
$$

with $a_{0}(t)=a_{n}(t)=1, a_{i}, q, g:\left[t_{0}, \infty\right) \rightarrow R, f: R \rightarrow R$ are continuous, $a_{i}(t)>0(i=1,2, \ldots, n-1), q(t)$ nonnegative and not identically zero on any ray $\left[t^{*}, \infty\right), t^{*} \geq t_{0}$ and $g(t) \rightarrow \infty$ as $t \rightarrow \infty$. We assume that 
(2) $\int^{\infty} \frac{1}{\mu_{i}(s)} d s=\infty$, where $\mu_{i}(t)=\max _{t^{*} \leq s \leq t} a_{i}(s)$ for $t \geq t^{*} \geq t_{0}$ and, for $i=1,2, \ldots, n-1$,

$$
x f(x)>0 \text { and } f^{\prime}(x) \geq k>0 \text { for } x \neq 0 \quad\left(1=\frac{d}{d x}\right) \text {. }
$$

We further assume that there exists a real-valued function $\sigma \in C^{1}\left[\left[_{0}, \infty\right),(0, \infty)\right)$ such that

$$
\begin{aligned}
& \sigma(t) \leq \inf _{s \geq t}\{s, g(s)\}, \\
& \sigma^{\circ}(t)>0, \\
& \sigma(t) \rightarrow \infty \text { as } t \rightarrow \infty .
\end{aligned}
$$

The domain $D\left(L_{n}\right)$ of $L_{n}$ is defined to be the set of all functions $x:\left[t_{0}, \infty\right) \rightarrow R$ such that $L_{j} x(t), 0 \leq j \leq n$, exist and are continuous on $\left[t_{0}, \infty\right)$. By a solution of (1) we mean a function $x \in D\left(L_{n}\right)$ which satisfies (1) on $\left[t_{0}, \infty\right)$. A nontrivial solution of (1) is called oscillatory if the set of its zeros is unbounded and it is called nonoscillatory otherwise.

The following lemma generalizes a well-known Kiguradze's lemma and can be proved similarly.

LEMMA 1. Let condition (2) hold and let $x \in D\left(L_{n}\right)$ be a positive fronction. If $L_{n} x(t)$ is of constant sign and not identically zero for all large $t$, then there exist $t_{x} \geq t_{0}$ and an integer $\tau, 0 \leq l \leq n$, with $n+l$ even for $L_{n} x$ nonnegative or $n+l$ odd for $L_{n} x$ nonpositive and such that, for every $t \geq t_{x}$,

$$
\imath>0 \text { implies } L_{k} x(t)>0(k=0,1, \ldots, 2-1)
$$

and

$$
\tau \leq n-1 \text { implies }(-1)^{2+k} L_{k} x(t)>0 \quad(k=\imath, 2+1, \ldots, n-1) .
$$

The following lemma appears in [3] and is needed in the sequel. 
LEMMA 2. Let $x \in D\left(L_{n}\right)$ with $x(t)>0$ for $t \geq t_{0}$. If

$$
L_{n-1} x(t) L_{n} x(t) \leq 0 \text { for all } t \geq t_{1} \geq t_{0} \text {. }
$$

$t_{1}$ is sufficiently large, then there exist $T \geq t_{1}$ and a positive constant $M$ such that, for each $t \geq T$,

$$
x \cdot(t) \geq \frac{M}{\mu_{1}(t)} \omega(T, \mu, t)\left|L_{n-1} x(t)\right|,
$$

where

$$
\omega(T, \mu, t)=\int_{T}^{t} \frac{1}{\mu_{1}\left(s_{2}\right)} \int_{T}^{s} \cdots \int_{T}^{s}{ }^{n-2} \frac{1}{\mu_{n-1}\left(s_{n-1}\right)} d s_{n-1} \cdots d s_{2} .
$$

THEOREM 1. Let conditions (2)-(4) hold. Suppose that there exists a continuously differentiable fronction

$$
\rho:\left[t_{0}, \infty\right) \rightarrow(0, \infty)
$$

such that for all sufficiently large $T$ with $\sigma\left(T_{1}\right)>T$ for some $T_{1}>T$ we have

$$
\lim _{t \rightarrow \infty} \sup \int_{T_{1}}^{t}\left[\rho(s) q(s)-\frac{\mu_{1}[\sigma(s)] \dot{\rho}^{2}(s)}{4 M k \rho(s) \dot{\sigma}(s) \omega(T, \mu, \sigma[s])}\right] d s=\infty,
$$

where $M$ is as in Lemma 2. Then every solution of (1) is oscillatory.

Proof. Let $x(t)$ be a nonoscillatory solution of (1). Assume that $x(t)>0$ for $t \geq t_{1} \geq t_{0}$, and choose a $t_{2} \geq t_{1}$ so that $\sigma(t) \geq t_{1}$ for $t \geq t_{2}$ and $x[\sigma(t)]>0$ for $t \geq t_{2}$. By Lemma 1 , there exists a. $t_{3} \geq t_{2}$ such that

$$
\dot{x}^{\circ}(t)>0 \text { and } L_{n-1} x(t)>0 \text { for all } t \geq t_{3} .
$$

Notice next that the hypotheses of Lemma 2 are satisfied on $\left[t_{3}, \infty\right)$ which implies that there exists a $t_{4} \geq t_{3}$ and a positive constant $M$ so that

$$
\dot{x}^{\circ}(t) \geq \frac{M}{\mu_{1}(t)} \omega\left(t_{4}, \mu, t\right) L_{n-1} x(t) \text { for all } t \geq t_{4} .
$$


Choose $t_{5} \geq t_{4}$ so that $\sigma(t)>t_{4}$ for all $t \geq t_{5}$. Thus

$$
\begin{aligned}
\dot{x}[\sigma(t)] & \geq \frac{M}{\mu_{1}[\sigma(t)]} \omega\left(t_{4}, \mu, \sigma(t)\right) L_{n-1} x[\sigma(t)] \\
& \geq \frac{M}{\mu_{1}[\sigma(t)]} \omega\left(t_{4}, \mu, \sigma(t)\right] L_{n-1} x(t) \text {, for } t \geq t_{5} .
\end{aligned}
$$

Let $W(t)=\rho(t) L_{n-1} x(t) / f(x[\sigma(t)])$. Thus $W(t)$ satisfies

$$
\dot{W}(t)=-\rho(t) q(t) \frac{f(x[g(t)])}{f(x[\sigma(t)])}+\frac{\dot{\rho}(t)}{\rho(t)} W(t)-\frac{\dot{\sigma}(t) \dot{x}[\sigma(t)] f^{\prime}(x[\sigma(t)]]}{f(x[\sigma(t)])} W(t) .
$$

From (6) and (7) we have

(8) $\dot{W}(t) \leq-\rho(t) q(t)+\frac{\dot{\rho}(t)}{\rho(t)} w(t)-\frac{M k \omega\left(t_{4}, \mu, \sigma(t)\right) \dot{\sigma}(t)}{\mu_{1}[\sigma(t)] \rho(t)} w^{2}(t)$

$$
\begin{aligned}
=-\rho(t) q(t)+\frac{\mu_{1}[\sigma(t)] \dot{\rho}^{2}(t)}{4 M k \rho(t) \dot{\sigma}(t) \omega\left(t_{4}, \mu, \sigma(t)\right)} \\
-\left[\frac{\left(M k \omega\left(t_{4}, \mu, \sigma(t)\right) \dot{\sigma}(t)\right)^{\frac{1}{2}}}{\mu_{1}[\sigma(t)] \rho(t)}\right)^{(t)} \\
\left.-\frac{\dot{\rho}(t) / \rho(t)}{2\left(M k \omega\left(t_{4}, \mu, \sigma(t)\right) \dot{\sigma}(t) / \mu_{1}[\sigma(t)] \rho(t)\right)^{\frac{1}{2}}}\right]^{2} .
\end{aligned}
$$

Thus

$$
\dot{W}(t) \leq-\rho(t) q(t)+\frac{\mu_{1}[\sigma(t)] \dot{p}^{2}(t)}{4 M k \rho(t) \dot{\sigma}(t) \omega\left(t_{4}, \mu, \sigma(t)\right]}
$$

Integrating (9) from $t_{5}$ to $t$ we obtain

$$
\begin{aligned}
\int_{t_{5}}^{t}\left[\rho(s) q(s)-\frac{\mu_{7}[\sigma(s)] \dot{\rho}^{2}(s)}{4 M k \rho(s) \dot{\sigma}(s) \omega\left(t_{4}, \mu, \sigma(s)\right)}\right] d s & \leq w\left(t_{5}\right)-w(t) \\
& \leq w\left(t_{5}\right)<\infty,
\end{aligned}
$$

which contradicts (5) and the proof of the theorem is complete.

THEOREM 2. Let conditions (3) and (5) of Theorem I be replaced by 


$$
\frac{f(x)}{x} \geq \gamma>0 \text { for } x \neq 0
$$

and

$$
\lim _{t \rightarrow \infty} \sup _{T_{1}}^{t}\left[\gamma \rho(s) q(s)-\frac{\mu_{1}[\sigma(s)] \dot{\rho}^{2}(s)}{4 \dot{\sigma} \dot{\sigma}(s) \rho(s) \omega(T, \mu, \sigma(s))}\right] d s=\infty,
$$

respectively. Then the conclusion of Theorem I holds.

Proof. The proof of Theorem 2 is similar to that of Theorem 1 except that we let $W(t)=\rho(t) L_{n-1} x(t) / x[\sigma(t)]$, and hence is omitted.

COROLLARY 1. In Theorem 1 (respectively Theorem 2), let conditions (5) (respectively (11)) be replaced by

$$
\lim _{t \rightarrow \infty} \sup \int_{T_{1}}^{t} \rho(s) q(s) d s=\infty
$$

and

$$
\lim _{t \rightarrow \infty} \sup \int_{T_{1}}^{\infty} \frac{\mu_{1}[\sigma(s)] \dot{\rho}^{2}(s)}{\rho(s) \dot{\sigma}(s) \omega(T, \mu, \sigma(s))} d s<\infty .
$$

Then the conclusion of Theorem 1 (respectively Theorem 2) holds.

THEOREM 3. Let conditions (2)-(4) hold, $m$ be an integer with $m \geq 3$ and $\rho$ be a positive continuously differentiable function on the interval $\left[t_{0}, \infty\right]$ such that

$$
\text { (14) } \begin{aligned}
\lim _{t \rightarrow \infty} \sup & \frac{1}{t^{m-1}} \int_{T_{1}}^{t}(t-s)^{m-3} \\
& {\left[(t-s)^{2} \rho(s) q(s)-\frac{[(t-s) \dot{\rho}(s)-(m-1) \rho(s)]^{2} \mu_{1}[\sigma(s)]}{4 M k \rho(s) \dot{\sigma}(s) \omega(T, \mu, \sigma(s))}\right] d s=\infty, }
\end{aligned}
$$

for all large $T$ with $\sigma\left(T_{1}\right)>T$ for some $T_{1}>T$, where $M$ is as in Lemma 2. Then equation (1) is oscillatory.

Proof. Let $x(t)$ be a non-oscillatory solution of (1), say $x(t)>0$ for $t \geq t_{1} \geq t_{0}$. As in the proof of Theorem 1, we obtain (8). Now we 
multiply both sides of (8) by $(t-s)^{m-1}$ and integrate from $t_{5}$ to $t$ to obtain

$$
\begin{aligned}
& \int_{t_{5}}^{t}(t-s)^{m-1} p(s) q(s) d s \\
& \leq\left(t-t_{5}\right)^{m-1} W\left(t_{5}\right)+\int_{t_{5}}^{t}(t-s)^{m-2}\left[(t-s) \frac{\dot{\rho}(s)}{\rho(s)}-(m-1)\right] W(s) d s \\
& -\int_{t_{5}}^{t}(t-s)^{m-1} \frac{M k \omega\left(t_{4}, \mu, \sigma(s)\right) \dot{\sigma}(s)}{\mu_{1}(\sigma(s)) \rho(s)} w^{2}(s) d s \\
& =\left(t-t_{5}\right)^{m-1} W\left(t_{5}\right) \\
& +\int_{t_{5}}^{t} \frac{(t-s)^{m-3}[(t-s) \dot{\rho}(s)-(m-1) \rho(s)]^{2} \mu_{1}[\sigma(s)]}{4 M k \rho(s) \dot{\sigma}(s) \omega\left(t_{4}, \mu, \sigma(s)\right)} d s \\
& -\int_{t_{5}}^{t}\left[\left[\frac{M k(t-s)^{m-1} \omega\left(t_{4}, \mu, \sigma(s)\right) \dot{\sigma}(s)}{\mu_{1}[\sigma(s)] \rho(s)}\right]^{\frac{1}{2}} W(s)\right. \\
& \left.-\frac{(t-s)^{m-2}[(t-s)(\dot{\rho}(s) / \rho(s))-(m-1)]}{2\left[\left(M k(t-s)^{m-1} \omega\left(t_{4}, \mu, \sigma(s)\right) \dot{\sigma}(s)\right) / \mu_{1}[\sigma(s)] \rho(s)\right]^{\frac{1}{2}}}\right]^{2} d s \text {. }
\end{aligned}
$$

Thus

$$
\begin{aligned}
\frac{1}{t^{m-1}} \int_{t_{5}}^{t}(t-s)^{m-3}\left[(t-s)^{2} \rho(s) q(s)\right. & \left.-\frac{[(t-s) \dot{\rho}(s)-(m-1) \rho(s)]^{2} \mu_{1}[\sigma(s)]}{4 M k \rho(s) \dot{\sigma}(s) \omega(t, \mu, \sigma(s))}\right] d s \\
& \leq 1-\left(t_{5} / t\right)^{m-1} \dot{w}\left(t_{5}\right) \rightarrow w\left(t_{5}\right)<\infty \text { as } t \rightarrow \infty,
\end{aligned}
$$

which contradict (14). A similar proof holds if $x(t)<0$ for $t \geq t_{1} \geq t_{0}$.

THEOREM 4. In Theorem 3, let conditions (3) and (14) be replaced respectively by condition (10) and 
(15) $\lim _{t \rightarrow \infty} \sup \frac{1}{t^{m-1}} \int_{T_{1}}^{t}(t-8)^{m-3}$

$$
\text { - }\left[\gamma(t-s)^{2} \rho(s) q(s)-\frac{[(t-s) \dot{\rho}(s)-(m-1) \rho(s)]^{2} \mu_{1}[\sigma(s)]}{4 M \rho(s) \dot{\sigma}(s) \omega(t, \mu, \sigma(s))}\right] d s=\infty \text {, }
$$

then the conclusion of Theorem 3 holds.

Proof. The proof of Theorem 4 is similar to that of Theorem 3 except that we let $W(t)=\rho(t) L_{n-1} x(t) / x[\sigma(t)]$, and hence we omit it.

COROLLARY 2. In Theorem 3 (respectively Theorem 4), let condition (4) (respectively (11)) be replaced by.

$$
\lim _{t \rightarrow \infty} \sup \frac{1}{t^{m-1}} \int_{T_{1}}^{t}(t-s)^{m-1} \rho(s) q(s) d s=\infty
$$

and

$$
\lim _{t \rightarrow \infty} \sup \frac{1}{t^{m-1}} \int_{T_{1}}^{t} \frac{[(t-s) \dot{\rho}(s)-(m-1) \rho(s)]^{2} \mu_{1}[\sigma(s)]}{\rho(s) \dot{\sigma}(s) \omega(T, \mu, \sigma(s))} d s<\infty .
$$

Then the conclusion of Theorem I (respectively Theorem 2) holds.

For illustration we consider the following examples.

EXAMPLE 1. The differential equation

$$
\left(\frac{1}{t}\left(\frac{1}{t}((1 / t) \dot{x})^{\cdot}\right)^{\bullet}\right)^{\cdot}+\frac{231}{16 t^{7}} x(t)=0, t \geq 1 \text {, }
$$

has the nonoscillatory solution $x(t)=\sqrt{t}$. Only condition (5) of Theorem 1 is violated.

EXAMPLE 2. Consider the equation

$$
\left(\frac{1}{t}\left(\frac{1}{t}((1 / t) \dot{x})^{\circ}\right)^{\circ}\right)^{\bullet}+\frac{1}{t^{3}} x[g(t)] \exp (\sin x[g(t)]\}=0, \quad t \geq 1,
$$

$g(t)=c t$ or $t \pm \sin t$. All conditions of Theorem 2 and Theorem 4 are satisfied for $\rho(t)=t^{2}$ and $\sigma(t)=c t, 0<c \leq 1$ for the first case and $\sigma(t)=t-1$ for the second. Hence all solutions of (19) are oscillatory. 
EXAMPLE 3. Consider the equation

$$
\left(t(t \ddot{x})^{\bullet}\right)^{\bullet}+\frac{1}{t^{2}} x[g(t)]=0, t \geq 1,
$$

where $g(t)=c t$ or $g(t)=t^{c}$ or $g(t)=t \pm c \cos t, c>0$. All conditions of Corollaries 1 and 2 are satisfied for $\rho(t)=t, \sigma(t) \leq c t$ or $t^{c}, 0<c \leq 1$ or $t-c$ (respectively for the three cases) and $m=3$, and hence all solutions of (20) are oscillatory. We note that Theorem 1 in [5] can be applied to (20), however in [5] only bounded solutions of (20) are discussed. Thus our results are more general than those in [5].

REMARK 1. The main results of Kamenev [4], Philos [6, 1], wintner [8] and Yeh [9, 10] are included in our Corollary 2 , for $n=2$ and $g(t)=t$. Those criteria are not applicable to equations of the form

$$
\ddot{x}+\frac{k^{2}}{t^{2}} x=0, k>\frac{1}{2},
$$

however Theorems 1 and 2 can be applied. Hence our results are a substantial improvement on the above mentioned results.

REMARK 2. The results obtained here are new, and we do not stipulate that the function $g$ in equation ( 1 ) is either retarded or advanced. Hence our theorems hold for ordinary, retarded, advanced, and mixed type equations.

\section{References}

[1] S.R. Grace and B.S. Lalli, "Oscillation theorems for $n$th order nonlinear functional differential equations", J. Math. Anal. App 2. 94 (1983), 509-524.

[2] S.R. Grace and B.S. Lalli, "An oscillation criterion for $n$th order nonlinear differential equations with functional arguments", Canad. Math. Buzl. 26 (1983), 35-40.

[3] S.R. Grace and B.S. Lalli, "Oscillatory and asymptotic behavior of solutions of differential equations with deviating arguments", J. Math. Anal. Appl. (to appear). 
[4] I.V. Kamenev, "An integral criterion for oscillation of linear differential equations of second order", Mat. Zametki 23 (1978), 249-251.

[5] D.L. Lovelady, "On oscillatory behavior of bounded solutions of higher order differential equations", $J$. Differential Equations 19 (1975), 167-175.

[6] Ch.G. Philos, "On a Kamenev's integral criterion for oscillation of linear differential equations of second order", Utilitas Math. 24 (1983), 277-289.

[7] Ch.G. Philos, "Oscillation of second order linear ordinary differential equations with alternating coefficients", Bull. Austral. Math. Soc. 27 (1983), 307-313.

[8] A. Wintner, "A criterion of oscillatory stability", Quart. Appl. Math. 7 (1949), 115-117.

[9] C.C. Yeh, "An oscillation criterion for second order nonlinear differential equations with functional arguments", J. Math. Anal. Appl. 76 (1980), 72-76.

[10] C.C. Yeh, "Oscillation theorems for nonlinear second order differential equations with damped term", Proc. Amer. Math. Soc. 84 (1982), 397-402.

Faculty of Engineering,

Cairo University,

Cairo,

Egypt;

Department of Mathematics, University of Saskatchewan, Saskatoon, Saskatchewan S7N OWO, Canada. 